\title{
Optimal Number of Cognitive Users in $K$-out-of- $M$ Rule
}

\author{
Narasimha Rao Banavathu, Student Member, IEEE and Mohammed Zafar Ali Khan, Senior Member, IEEE
}

\begin{abstract}
In this letter, we obtain a generalized expression for the optimal number of cognitive users (CUs) for the $K$-out-of$M$ rule that minimizes the Bayes risk at the fusion center (FC) over noisy control channels. We show many existing and new are special cases of the proposed solution. Numerical results are presented using energy detector. However, the expressions for optimal $M$ obtained in this letter are applicable to any detector used in cooperative spectrum sensing.
\end{abstract}

Index Terms-Cognitive radio, Bayes risk function, noisy control channel, number of cognitive users.

\section{INTRODUCTION}

Cognitive radio (CR) has been proposed to overcome the spectrum shortage problem and the spectrum underutilization of current radio spectrum by allowing the cognitive users (CUs) to access spectrum of the licensed or primary user (PU) under sufficient protection to the PU [1], [2]. To do so, the CUs must continuously sense to identify the free spectrum and must be able to detect the presence of the PU signal [3]. Well-known detectors such as conventional energy detector (ED) [4], improved energy detector (IED) [5], etc., have been studied to determine the presence of the PU.

However, spectrum sensing using one CU may results in poor detection performance due to multipath and shadowing and may results in interference to the PU. In order to improve the reliability in detecting the PU signal, cooperative spectrum sensing (CSS) [6], [7] can be employed. The idea of CSS is to use multiple CUs and combine their observations at the fusion center (FC) using $K$-out-of- $M$ rule [8]. The $K$-out-of$M$ rule decides the presence of PU if at least $K$ out of $M$ CUs must detect the PU signal. However, in practice, the control channels between the CUs and the FC are noisy [9], [10]. This will deteriorate the reliability of decisions transmitted from the CUs to the FC. A detailed survey on spectrum sensing and CSS which also highlights the research challenges and unsolved problems are presented in [11], [12].

Most of the recent work focuses on optimizing $K$ of the $K$-out-of- $M$ rule aiming for different objectives such as minimizing the Bayes risk function [8, p. 94], minimizing the total error rate [13], [14], maximizing the energy efficiency [15]. In [16], optimal $K$ is found to maximize the CUs network throughput while satisfying protection constraint to the PU. In [17], an algorithm for the optimal $K$ is presented to maximize the global detection probability subject to a constraint on global false alarm probability. In [18], the optimal $K$ and the

Narasimha Rao Banavathu and Mohammed Zafar Ali Khan are with the Department of Electrical Engineering, Indian Institute of Technology Hyderabad, 502285, India (e-mail: ee13p1005@iith.ac.in; zafar@iith.ac.in).

This research work was partly sponsored by YFRF, MeitY. optimal detection threshold of the multi-hop CR network are derived. Some studies on optimizing the $M$ are as follows; the optimum value of $M$ that minimizes the total error rate for (i) OR rule is obtained in [19], [20] (ii) for AND and (iii) MAJORITY rule in [21]. In [15], the optimal $M$ to maximize the energy efficiency is obtained through an exhaustive search algorithm. In this letter, we formulate the general optimization problem (GOP) for finding the optimal $M$ that minimizes the Bayes risk function and then we show that most existing works [19]-[22] are special cases of GOP. Finally, we present the solutions for GOP and its special cases.

The outline of this paper is as follows. In Section II, we describe the system model for the CSS. In Section III, we formulate the GOP and its spacial cases for finding the optimal $M$. In Section IV, we present the solutions for the formulated problems. Section-V presents the numerical results using energy detector followed by conclusions in Section VI.

\section{SySTEM MODEL}

We consider a centralized CSS model [14] composed of $M$ CUs, a PU and a FC. Each CU conducts the spectrum sensing over the sensing channel and makes a binary decision regarding the presence of PU. Let $\mathcal{H}_{0}$ and $\mathcal{H}_{1}$ denote the hypotheses for the absence and presence of the PU, respectively. The local false alarm and missed detection probabilities of the $k$ th $\mathrm{CU}$ are given, respectively, by

$$
P_{f}^{k}=\operatorname{Pr}\left\{d_{k}=1 \mid \mathcal{H}_{0}\right\}, P_{m}^{k}=\operatorname{Pr}\left\{d_{k}=0 \mid \mathcal{H}_{1}\right\},
$$

where $d_{k} \in\{0,1\}$ is the binary decision of the $k$ th $\mathrm{CU}$ indicating the hypotheses $\mathcal{H}_{0}$ and $\mathcal{H}_{1}$, respectively. The local decisions from $M$ CUs are transmitted to the FC over noisy control channels. The FC makes the final decision on the status of the PU by adopting the $K$-out-of- $M$ rule [8]. Following [13], we assume that the CR network is homogenous, this implies $P_{f}^{k}=P_{f}, P_{m}^{k}=P_{m}, \forall k$. Let $P_{e}^{k}$ denote the error probability of a control channel between the $k$ th $\mathrm{CU}$ and the FC. We assume that all control channels are identical, which implies $P_{e}^{k}=P_{e}$. Under these assumptions, the global false alarm and missed detection probabilities for the $K$-out-of- $M$ rule are given, respectively, by [10]

$$
P_{F}(K, M)=\operatorname{Pr}\left(D=1 \mid \mathcal{H}_{0}\right)=\sum_{i=K}^{M}\left(\begin{array}{c}
M \\
i
\end{array}\right) P_{f e}^{i}\left(1-P_{f e}\right)^{M-i}
$$

$$
\begin{aligned}
P_{M}(K, M) & =\operatorname{Pr}\left(D=0 \mid \mathcal{H}_{1}\right) \\
& =1-\sum_{i=K}^{M}\left(\begin{array}{c}
M \\
i
\end{array}\right)\left(1-P_{m e}\right)^{i} P_{m e}^{M-i},
\end{aligned}
$$


where $D \in\{0,1\}$ is the final decision drawn by the FC that the PU is absent and present, respectively and

$$
\begin{aligned}
P_{f e} & =P_{f}\left(1-P_{e}\right)+\left(1-P_{f}\right) P_{e}, \\
P_{m e} & =P_{m}\left(1-P_{e}\right)+\left(1-P_{m}\right) P_{e} .
\end{aligned}
$$

The Bayes risk function for the $K$-out-of- $M$ rule is given by $[8$, eq. 2.2 .1$]$

$$
\begin{aligned}
\mathcal{R}(K, M) & =\sum_{m=0}^{1} \sum_{n=0}^{1} \beta_{m n} P_{n} \operatorname{Pr}\left(D=m \mid \mathcal{H}_{n}\right) \\
& =\beta_{F} P_{F}(K, M)+\beta_{M} P_{M}(K, M)+\beta_{C},(5)
\end{aligned}
$$

where $\beta_{F}=P_{0}\left(\beta_{10}-\beta_{00}\right), \beta_{M}=P_{1}\left(\beta_{01}-\beta_{11}\right)$ and $\beta_{C}=$ $\beta_{00} P_{0}+\beta_{11} P_{1} . \beta_{m n}, \forall m, n \in\{0,1\}$, is the cost incurred by declaring the final decision $D=m$ by the FC when the true hypothesis about the PU is $\mathcal{H}_{n}$, and where $P_{0}$ and $P_{1}$ denote the $a$ priori probabilities of the PU being absent and present, respectively.

\section{PRoblem Formulation}

The general optimization problem (GOP) can now be formulated as

$$
\begin{array}{cl}
\underset{M}{\operatorname{minimize}} & \mathcal{R}(K, M) \\
\text { subject to } & \mathbb{C}_{1}: P_{f e}=P_{f e}^{0}, \\
& \mathbb{C}_{2}: P_{m e}=P_{m e}^{0}, \\
& \mathbb{C}_{3}: K=K_{0},
\end{array}
$$

where $\mathcal{R}(K, M)$ is given in (5). $P_{f e}^{0}, P_{m e}^{0}$ and $K_{0}$ are the equality constraints.

\section{A. Special Cases of GOP}

Now we present special cases of the GOP.

- GOP-I: Substituting $\beta_{10}=\beta_{01}=1$ and $\beta_{00}=\beta_{11}=$ 0 in (6), we get the optimization problem for finding the optimal $M$ that minimizes the average probability of error of the $K$-out-of- $M$ rule.

- GOP-II: Substituting $\beta_{10}=\beta_{01}=2, \beta_{00}=\beta_{11}=0$ and $P_{0}=P_{1}=0.5$ in (6), we get the optimization problem for finding the optimal $M$ that minimizes the total error rate of the $K$-out-of- $M$ rule [22].

- GOP-III: Substituting $\beta_{10}=\beta_{01}=2, \beta_{00}=\beta_{11}=0$, $P_{0}=P_{1}=0.5$ and $K=1$ in (6), we get the optimization problem for finding the optimal $M$ that minimizes the total error rate for the OR rule [19], [20].

- GOP-IV: Substituting $\beta_{10}=\beta_{01}=2, \beta_{00}=\beta_{11}=0$, $P_{0}=P_{1}=0.5$ and $K=M$ in (6), we get the optimization problem for finding the optimal $M$ that minimizes the total error rate for the AND rule [21].

- GOP-V: Substituting $\beta_{10}=\beta_{01}=2, \beta_{00}=\beta_{11}=0$, $P_{0}=P_{1}=0.5$ and $K=\lceil M / 2\rceil$ in (6), we get the optimization problem for finding the optimal $M$ that minimizes the total error rate of the MAJORITY rule [21].

Note that GOP-II to GOP-V have been addressed in literature while GOP-I is a new optimization problem. Also note that the minimization of total error rate and average probability of error result in different optimal solutions.

\section{SOlution OF THE GOP}

Theorem 1. The solution for GOP in (6), denoted as $M_{\mathcal{R}}^{*}$, is given by

$$
M_{\mathcal{R}}^{*}=\left\lceil K \beta+K-1+\frac{\ln \frac{P_{1}\left(\beta_{01}-\beta_{11}\right)}{P_{0}\left(\beta_{10}-\beta_{00}\right)}}{\ln \frac{1-P_{f e}}{P_{m e}}}\right\rceil, \beta=\frac{\ln \frac{1-P_{m e}}{P_{f e}}}{\ln \frac{1-P_{f e}}{P_{m e}}},
$$

where $\lceil$.$\rceil represents the ceiling function and P_{f e}, P_{m e}$ are given by (3), (4) respectively.

Proof: The optimal $M$ that satisfies (6) can be obtained by setting the difference of $\mathcal{R}$ with respect to $M$ and equating to zero. i.e,

$$
\begin{gathered}
\mathcal{R}(K, M+1)-\mathcal{R}(K, M)=0 . \\
\Rightarrow \beta_{F}\left(P_{F}(K, M+1)-P_{F}(K, M)\right)+ \\
\beta_{M}\left(P_{M}(K, M+1)-P_{M}(K, M)\right)=0 .
\end{gathered}
$$

To further simplify (8), we consider a function $\mathcal{F}$ in terms of $P(K, M)=\sum_{i=K}^{M}\left(\begin{array}{c}M \\ i\end{array}\right) \alpha^{i}(1-\alpha)^{M-i}$ which is given by

$$
\begin{aligned}
& \mathcal{F}(M)=P(K, M+1)-P(K, M) \\
= & \sum_{i=K}^{M+1}\left(\begin{array}{c}
M+1 \\
i
\end{array}\right) \alpha^{i}(1-\alpha)^{M-i+1}-\sum_{i=K}^{M}\left(\begin{array}{c}
M \\
i
\end{array}\right) \alpha^{i}(1-\alpha)^{M-i} \\
= & \alpha^{M+1}+ \\
& \sum_{i=K}^{M}\left[\left(\begin{array}{c}
M+1 \\
i
\end{array}\right) \alpha^{i}(1-\alpha)^{M-i+1}-\left(\begin{array}{c}
M \\
i
\end{array}\right) \alpha^{i}(1-\alpha)^{M-i}\right] .
\end{aligned}
$$

Substituting $\left(\begin{array}{c}M+1 \\ i\end{array}\right)=\left(\begin{array}{c}M \\ i\end{array}\right)+\left(\begin{array}{c}M \\ i-1\end{array}\right)$ into (9) and rearranging, we have

$$
\begin{gathered}
\mathcal{F}(M)=\alpha^{M+1}+\sum_{i=K}^{M}\left(\begin{array}{c}
M \\
i-1
\end{array}\right) \alpha^{i}(1-\alpha)^{M-i+1}+ \\
\sum_{i=K}^{M}\left(\begin{array}{c}
M \\
i
\end{array}\right) \alpha^{i}\left[(1-\alpha)^{M+1-i}-(1-\alpha)^{M-i}\right] \\
=\alpha^{M+1}+\sum_{i=K}^{M}\left(\begin{array}{c}
M \\
i-1
\end{array}\right) \alpha^{i}(1-\alpha)^{M-i+1} \\
-\sum_{i=K}^{M}\left(\begin{array}{c}
M \\
i
\end{array}\right) \alpha^{i+1}(1-\alpha)^{M-i} .
\end{gathered}
$$

By expanding the summation terms in the above equation, we have

$$
\begin{aligned}
& \mathcal{F}(M)=\alpha^{M+1}+\left(\begin{array}{c}
M \\
K-1
\end{array}\right) \alpha^{K}(1-\alpha)^{M-K+1} \\
&+\left(\begin{array}{c}
M \\
K
\end{array}\right) \alpha^{K+1}(1-\alpha)^{M-K}+\ldots+\left(\begin{array}{c}
M \\
M-1
\end{array}\right) \alpha^{M}(1-\alpha) \\
&-\left(\begin{array}{c}
M \\
K
\end{array}\right) \alpha^{K+1}(1-\alpha)^{M-K}-\ldots .-\left(\begin{array}{c}
M \\
M-1
\end{array}\right) \alpha^{M}(1-\alpha) \\
& \quad-\left(\begin{array}{l}
M \\
M
\end{array}\right) \alpha^{M+1}
\end{aligned}
$$




$$
=\left(\begin{array}{c}
M \\
K-1
\end{array}\right) \alpha^{K}(1-\alpha)^{M-K+1} .
$$

By substituting (10) into (8), we have

$$
\beta_{F} P_{f e}^{K}\left(1-P_{f e}\right)^{M-K+1}-\beta_{M}\left(1-P_{m e}\right)^{K} P_{m e}^{M-K+1}=0 .
$$

Substituting $\beta_{F}=P_{0}\left(\beta_{10}-\beta_{00}\right)$ and $\beta_{M}=P_{1}\left(\beta_{01}-\beta_{11}\right)$ in the above equation and rearranging, we get

$$
M=K \beta+K-1+\frac{\ln \frac{P_{1}\left(\beta_{01}-\beta_{11}\right)}{P_{0}\left(\beta_{10}-\beta_{00}\right)}}{\ln \frac{1-P_{f e}}{P_{m e}}}, \beta=\frac{\ln \frac{1-P_{m e}}{P_{f e}}}{\ln \frac{1-P_{f e}}{P_{m e}}} .
$$

Since $M$ is an integer value, therefore we take ceiling function for the $M$ in (11) to obtain (7).

Note that (7) is a function of $P_{f}, P_{m}$ and $P_{e}$. Therefore, (7) is applicable to any detector used in the CSS. Now, we present the solution of optimal $M$ for the special cases of GOP as follows.

\section{A. Solutions for the Special Cases of GOP}

The solution for GOP-I, denoted as $M_{P_{E}}^{*}$ and can be obtained by direct substitution of $\beta_{10}=\beta_{01}=1, \beta_{00}=\beta_{11}=0$ in (11), we get $M_{P_{E}}^{*}=\left\lceil K \beta+K-1+\frac{\ln \left(P_{1} / P_{0}\right)}{\ln \frac{1-P_{f e}}{P_{m e}}}\right]$. The solution for GOP-II, denoted as $M_{P_{T}}^{*}$ and can be obtained by direct substitution of $\beta_{10}=\beta_{01}=2, \beta_{00}=\beta_{11}=0$ and $P_{0}=P_{1}=0.5$ in (11), we get [22] $M_{P_{T}}^{*}=\lceil K \beta+K-1\rceil$. The solution for GOP-III, denoted as $M_{P_{T}}^{O R}$ and can be obtained by direct substitution of $\beta_{10}=\beta_{01}=2, \beta_{00}=$ $\beta_{11}=0, P_{0}=P_{1}=0.5$ and $K=1$ in (11), we get [19], [20] $M_{P_{T}}^{O R}=\lceil\beta\rceil$. The solution for GOP-IV, denoted as $M_{P_{T}}^{A N D}$ and can be obtained by substituting $\beta_{10}=\beta_{01}=2$, $\beta_{00}=\beta_{11}=0, P_{0}=P_{1}=0.5$ and $K=M$ in (11) and rearranging, we get [21] $M_{P_{T}}^{A N D}=\lceil 1 / \beta\rceil$.

The solution for GOP-V is a bit involved and can be obtained by substituting $\beta_{10}=\beta_{01}=2, \beta_{00}=\beta_{11}=0$, $P_{0}=P_{1}=0.5$ and $K=\lceil M / 2\rceil$ in (11), we have

$$
\begin{aligned}
M & =\lceil M / 2\rceil \beta+\lceil M / 2\rceil-1 \\
& \Rightarrow\lceil M / 2\rceil=\frac{M+1}{\beta+1} .
\end{aligned}
$$

Using mathematical definition of ceiling function, above equation can be written as,

$$
\frac{M+1}{\beta+1}-1<\frac{M}{2} \leq \frac{M+1}{\beta+1} .
$$

Considering left hand side inequality of (12), we have

$$
\frac{M+1}{\beta+1}-1<\frac{M}{2}, \Rightarrow M<\frac{2 \beta}{1-\beta}
$$

Note that $M$ is non-negative integer value, therefore (13) is valid when $\beta<1$ which implies $P_{m e}<P_{f e}$. Considering right hand side inequality of (12), we have

$$
\frac{M}{2} \leq \frac{M+1}{\beta+1}, \Rightarrow M \leq 2 /(\beta-1) .
$$

Combining (13) and (14) we have [21]

$$
M_{P_{T}}^{M A J}= \begin{cases}\lceil 2 \beta /(1-\beta)\rceil, & P_{m e}<P_{f e}, \\ \lceil 2 /(\beta-1)\rceil, & P_{m e} \geq P_{f e} .\end{cases}
$$

\section{Numerical Results using Energy Detector}

We present the numerical results using energy detector (ED) as an example for analyzing our results obtained in this paper. The $P_{f}$ and $P_{m}$ of a $\mathrm{CU}$ using $\mathrm{ED}$ over additive white Gaussian noise (AWGN) channel are given, respectively, by [4]

$$
P_{f}=\frac{\Gamma\left(\mu, \frac{\lambda}{2}\right)}{\Gamma(\mu)}, P_{m}=1-Q_{\mu}(\sqrt{2 \gamma}, \sqrt{\lambda}),
$$

where the standard notations $\Gamma(.,),. \Gamma($.$) and Q_{\mu}(.,$.$) denotes$ the upper incomplete gamma function, gamma function and generalized Marcum $Q$-function of order $\mu-1$, respectively and $\mu$ denote the time-bandwidth product, $\lambda$ and $\gamma$ represents the sensing threshold and received signal-to-noise ratio (SNR) of a $\mathrm{CU}$, respectively. Note that, in (16) $P_{f}$ is a decreasing function and $P_{m}$ is a increasing function with $\lambda$, respectively.

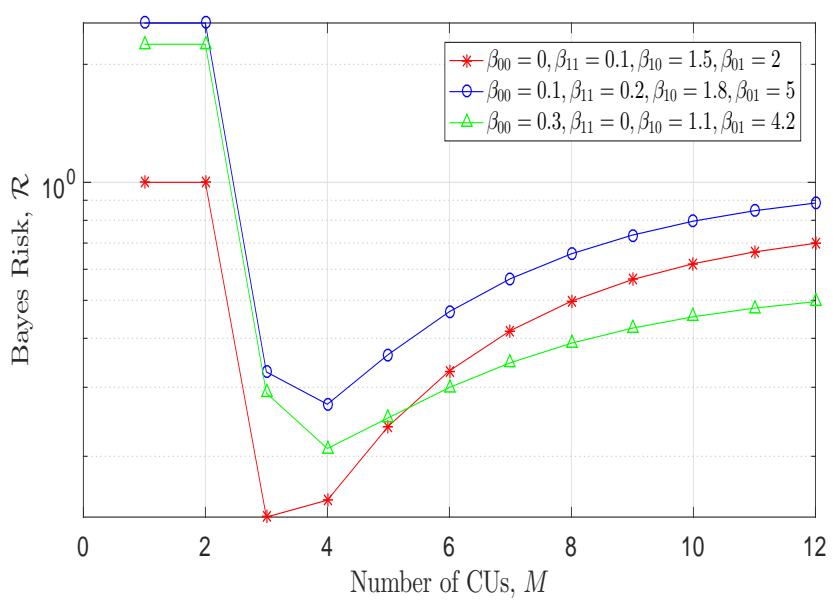

Fig. 1. Bayes risk versus Number CUs, $M$ for $P_{0}=P_{1}=0.5, \lambda=11$, $K=3, \mu=5, P_{e}=0.01$ and $\mathrm{SNR}=10 \mathrm{~dB}$, using ED

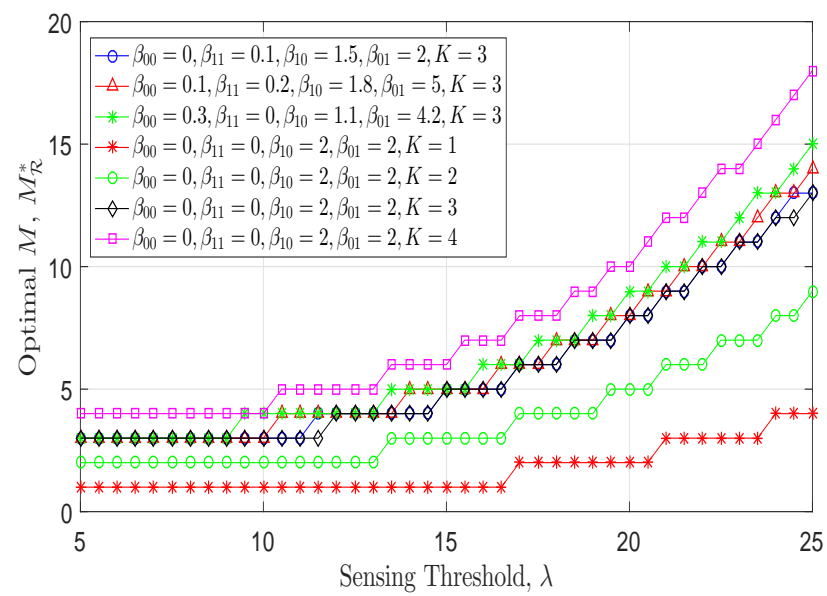

Fig. 2. Optimal $M$ versus sensing threshold, $\lambda$ for $P_{0}=P_{1}=0.5, \mu=5$, $P_{e}=0.01$ and $\mathrm{SNR}=10 \mathrm{~dB}$, using ED.

Fig. 1 plots the Bayes against number of CUs for various combination of cost values using ED. It can be noticed that, for 
each combination of cost values, the Bayes risk first decreases and then increases as $M$ increases which suggests an optimal value of $M$. Fig. 2 plots the solution of optimal $M$ versus sensing threshold. From Fig. 2, note that at $\lambda=16.8$, we get $P_{f e}=P_{m e}=0.08$ for $P_{e}=0.01$. Therefore, when $\lambda \leq 16.8$ implies $P_{m e} \leq P_{f e}$ and when $\lambda>16.8$ implies $P_{m e}>P_{f e}$. From Fig. 2, now we make the following observations for the case when $\beta_{00}=0, \beta_{11}=0, \beta_{01}=2, \beta_{10}=2$.

- OR Rule: Cooperation using OR rule is beneficial and there exists an optimal $M$ for $K=1$. This can can be achieved when $\lambda>16.8$, which implies $P_{m e}>P_{f e}$.

- AND Rule: Cooperation using AND rule is beneficial and there exists an optimal $M$ when $K=M_{\mathcal{R}}^{*}$. This can be achieved when $\lambda<16.8$, which implies $P_{m e}<P_{f e}$.

- MAJORITY Rule: Cooperation using MAJORITY rule is beneficial and there exists an optimal $M$ when $K=$ $\left\lceil M_{\mathcal{R}}^{*} / 2\right\rceil$. This can be achieved when $\lambda \approx 16.8$, which implies $P_{m e} \approx P_{f e}$ (where the relation between $P_{f e}$ and $P_{m e}$ can be $P_{m e} \geq P_{f e}$ or $\left.P_{m e}>P_{f e}\right)$.

Fig. 3 plots the optimal $M$ versus SNR for three values of $P_{e}$. It can be noticed that, the optimal $M$ decreases with SNR. For a fixed SNR the optimal $M$ decreases with $P_{e}$, because the error in the control channel reduces the reliability of decisions received at the $\mathrm{FC}$.

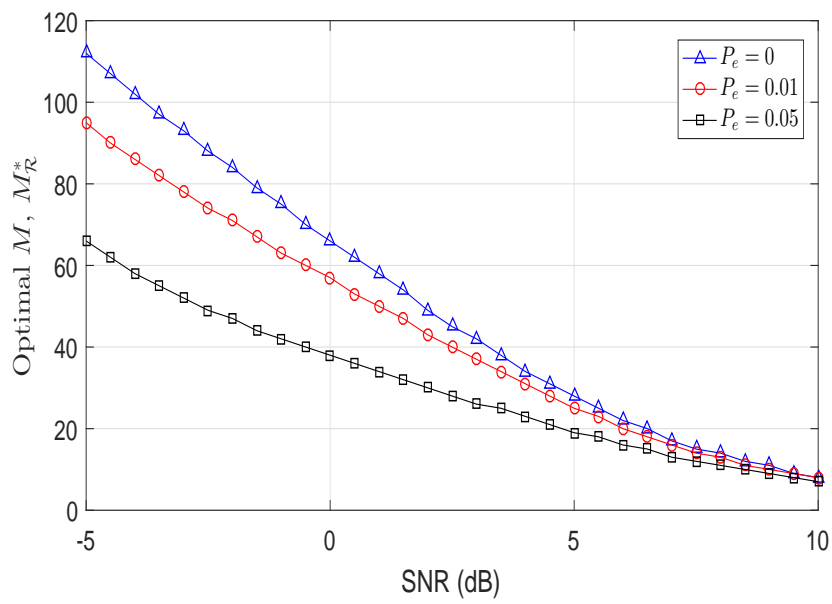

Fig. 3. Optimal $M$ versus SNR for $\beta_{00}=0.1, \beta_{11}=0.2, \beta_{10}=2, \beta_{01}=$ $3, P_{0}=P_{1}=0.5, \mu=5$ and $K=3$, using ED.

\section{CONCLUSIONS}

In this letter, we obtained a generalized expression for optimal number of CUs in the presence of control channel errors that minimizes the Bayes risk. We show that many existing results are special cases of the generalized expression. Also the results are valid for any detector.

\section{REFERENCES}

[1] J. Mitola and G. Q. Maguire, "Cognitive radio: making software radios more personal," IEEE Personal Communications, vol. 6, no. 4, pp. 1318, Aug 1999.

[2] S. Haykin, "Cognitive radio: brain-empowered wireless communications," Selected Areas in Communications, IEEE Journal on, vol. 23, no. 2 , pp. $201-220$, feb. 2005.
[3] E. G. Larsson and M. Skoglund, "Cognitive radio in a frequencyplanned environment: some basic limits," IEEE Transactions on Wireless Communications, vol. 7, no. 12, pp. 4800-4806, December 2008.

[4] F. F. Digham, M. S. Alouini, and M. K. Simon, "On the energy detection of unknown signals over fading channels," in Communications, 2003. ICC '03. IEEE International Conference on, vol. 5, May 2003, pp. 35753579 vol.5.

[5] Y. Chen, "Improved energy detector for random signals in gaussian noise," Wireless Communications, IEEE Transactions on, vol. 9, no. 2, pp. 558-563, February 2010.

[6] A. Ghasemi and E. S. Sousa, "Collaborative spectrum sensing for opportunistic access in fading environments," in First IEEE International Symposium on New Frontiers in Dynamic Spectrum Access Networks, 2005. DySPAN 2005., Nov 2005, pp. 131-136.

[7] K. Letaief and W. Zhang, "Cooperative communications for cognitive radio networks," Proceedings of the IEEE, vol. 97, no. 5, pp. 878-893, May 2009.

[8] P. K. Varshney, "Distributed detectiona and data fusion," Springer 1997.

[9] W. Zhang and K. B. Letaief, "Cooperative spectrum sensing with transmit and relay diversity in cognitive radio networks - [transaction letters]," IEEE Transactions on Wireless Communications, vol. 7, no. 12, pp. 4761-4766, December 2008.

[10] S. Chaudhari, J. LundÃl'n, V. Koivunen, and H. V. Poor, “ $\{B E P\}$ walls for cooperative sensing in cognitive radios using k-out-of-n fusion rules," Signal Processing, vol. 93, no. 7, pp. 1900 - 1908, 2013.

[11] I. F. Akyildiz, B. F. Lo, and R. Balakrishnan, "Cooperative spectrum sensing in cognitive radio networks: A survey," Physical Communication, vol. 4, no. 1, pp. $40-62,2011$.

[12] L. Lu, X. Zhou, U. Onunkwo, and G. Y. Li, "Ten years of research in spectrum sensing and sharing in cognitive radio," EURASIP Journal on Wireless Communications and Networking, vol. 2012, no. 1, p. 28 , 2012.

[13] W. Zhang, R. Mallik, and K. Letaief, "Optimization of cooperative spectrum sensing with energy detection in cognitive radio networks," Wireless Communications, IEEE Transactions on, vol. 8, no. 12, pp. $5761-5766$, december 2009.

[14] N. R. Banavathu and M. Z. A. Khan, "Optimal n-out-of- k voting rule for cooperative spectrum sensing with energy detector over erroneous control channel," in 2015 IEEE 81st Vehicular Technology Conference (VTC Spring), May 2015, pp. 1-5.

[15] H. Hu, H. Zhang, H. Yu, Y. Chen, and J. Jafarian, "Energy-efficient design of channel sensing in cognitive radio networks," Computers and Electrical Engineering, vol. 42, pp. 2017 - 220, 2015.

[16] E. C. Y. Peh, Y. C. Liang, Y. L. Guan, and Y. Zeng, "Optimization of cooperative sensing in cognitive radio networks: A sensing-throughput tradeoff view," IEEE Transactions on Vehicular Technology, vol. 58, no. 9, pp. 5294-5299, Nov 2009.

[17] S. Maleki, S. P. Chepuri, and G. Leus, "Optimal hard fusion strategies for cognitive radio networks," in 2011 IEEE Wireless Communications and Networking Conference, March 2011, pp. 1926-1931.

[18] A. Singh, M. R. Bhatnagar, and R. K. Mallik, "Performance of an improved energy detector in multihop cognitive radio networks," IEEE Transactions on Vehicular Technology, vol. 65, no. 2, pp. 732-743, Feb 2016.

[19] A. Singh, M. Bhatnagar, and R. Mallik, "Optimization of cooperative spectrum sensing with an improved energy detector over imperfect reporting channels," in Vehicular Technology Conference (VTC Fall), 2011 IEEE, Sept 2011, pp. 1-5.

[20] A. Singh, M. R. Bhatnagar, and R. K. Mallik, "Cooperative spectrum sensing in multiple antenna based cognitive radio network using an improved energy detector," IEEE Communications Letters, vol. 16, no. 1, pp. 64-67, January 2012.

[21] N. R. Banavathu and M. Z. A. Khan, "On cooperative spectrum sensing with improved energy detector over erroneous control channel," in 2016 IEEE Wireless Communications and Networking Conference, April 2016, pp. 1-6.

[22] — - "Optimization of cooperative spectrum sensing in cognitive radio over erroneous reporting channel," in Accepted in IEEE Wireless Personal Multimedia Communications Symposium, December 2015, pp. $1-5$. 